\title{
HPV DNA Plasmids Therapeutic Vaccine VGX-3100
}

National Cancer Institute

\section{Source}

National Cancer Institute. HPV DNA Plasmids Therapeutic Vaccine VGX-3100. NCI

Thesaurus. Code C77909.

\begin{abstract}
A DNA vaccine consisting of plasmids encoding the E6 and E7 genes of human papilloma virus (HPV) subtypes 16 and 18, respectively, with potential immunostimulating and antineoplastic activities. Administered via intramuscular electroporation, HPV DNA plasmids therapeutic vaccine VGX-3100 expresses E6 and E7 proteins, which may elicit a cytotoxic T-lymphocyte (CTL) response ag ainst cervical cancer cells expressing E6 and E7 proteins, resulting in tumor cell lysis. HPV type 16 and HPV type 18 are the most common HPV types involved in cervical carcinogenesis.
\end{abstract}

